Itinéraires Itinéraires

Littérature, textes, cultures

2011-3 | 2011

Récits du corps au Maroc et au Japon

\title{
Les bains japonais (湯) : un espace relationnel
}

\author{
Junko Komatsu
}

\section{OpenEdition}

\section{Journals}

Édition électronique

URL : http://journals.openedition.org/itineraires/1545

DOI : 10.4000/itineraires.1545

ISSN : 2427-920X

Éditeur

Pléiade

Édition imprimée

Date de publication : 1 novembre 2011

Pagination : $129-140$

ISBN : 978-2-296-55720-8

ISSN : 2100-1340

Référence électronique

Junko Komatsu, «Les bains japonais (湯) : un espace relationnel », Itinéraires [En ligne], 2011-3 | 2011, mis en ligne le 01 novembre 2011, consulté le 30 avril 2019. URL : http://journals.openedition.org/ itineraires/1545; DOI : 10.4000/itineraires.1545

Itinéraires est mis à disposition selon les termes de la licence Creative Commons Attribution - Pas d'Utilisation Commerciale - Pas de Modification 4.0 International. 


\title{
Les bains japonais (湯) : un espace relationnel
}

\begin{abstract}
Japanese baths (including thermal stations, or onsen, and public baths, or sentô) are very popular among Japanese people. We must make a difference between domestic bath and public bath. Public and thermal baths represent an important topic in visual arts, as well as for literature. It is one of the most stimulating frame and location for Japanese imaginative world. Apart from collective or individual enjoyment and purification, one of its functions is to contribute to a wider social mix and to another kind of verbal and non-verbal relation, called skinship. Last but not least, Japanese baths provide the user with a feeling of eternity.
\end{abstract}

Keywords : Japanese, thermal baths, public baths, space, skin ship Mots clés : Japonais, station thermale, bains publics, espace, relation par la peau

Les bains publics au Japon appartiennent encore à la culture populaire, et quoiqu'en nette régression, restent fréquentés dans les grandes villes, mais aussi dans les stations thermales, ou à la campagne. Il convient de prendre en compte cette réalité sociologique. Par ailleurs, ils sont un élément médiatisé par les arts visuels, un thème littéraire, mais aussi un cadre et un lieu stimulant pour l'imaginaire. Les bains sont aussi une réalité forte du monde arabe, connue notamment sous le nom de « hammam ». Abdelkébir Khatibi dialogue avec Tanizaki Junichirô dans une relation en miroir qui se lit dans les titres même de leurs ouvrages : Éloge de l'ombre pour l'un, Ombres japonaises pour l'autre'. Pourtant, il faut chercher dans d'autres ouvrages de ces auteurs pour y reconnaître ce thème. Les bains ne sont-ils pas, et c'est l'une de leurs fonctions, lieu de l'échange et du mélange? Nous voudrions établir ici un bref parcours de ce que les bains représentent au Japon, comme habitus, mais aussi comme contenant d'une représentation originale du corps, au sens où les écrivains indiquent à la fois

1. Tanizaki Junichirô, Éloge de l'ombre, trad. René Sieffert, Paris, Publications orientalistes de France [POF], 1978; Abdelkébir Khatibi, Ombres japonaises, Fontfroide le haut, Fata Morgana, 1988. 
une identité sociale et personnelle à travers leur description de cet espace de relation avec son propre corps et avec le corps des autres.

\section{Phénoménologie du bain japonais}

\section{Les noms du bain}

Le terme générique $y u$ (湯 ou $ゆ$ ) pour eau chaude renvoie aux possibilités naturelles du thermalisme (onsen, 温泉) pour des îles volcaniques, et à l'eau chauffée dans les bains publics nommés sentô (銭湯) ${ }^{2}$, ou furoya. C'est le signe cursif (ゆ) qui flotte sur le rideau souvent bleu à l'entrée, mais l'enseigne du bain est aussi bien un trait en forme de coupe d'où montent trois traits tortillés de vapeur (仙). On emploie la dénomination générale, o-furo (お風呂), et on dit 《entrer dans le bain», 《o-furo ni hairu » plutôt que « prendre un bain ». Le « vent» entre dans la composition des caractères écrits qui désignent le bain de façon plus précise, mais on peut penser aussi au sens de « pratique », ou de « manière de faire ».

\section{L'hygiène et la purification}

Il faut préciser que l'eau du bain se partage, chez soi, ou dans un établissement public, ce qui induit des règles précises. La revue Critique, titrée « Dans le bain japonais ${ }^{3}$ », s'ouvre sur un article faisant entrer directement dans le bain. Mizubayashi Akira y explique l'usage du bain privé à un étranger, tout comme l'auteur de mangas Suehiro Maruo dessine les étapes du bain dans un guide édité par le J.T.B. (Japan Travel Bureau). Dans tous les cas, il est fait mention de prescriptions contradictoires, et l'usage général comporte de sérieuses entorses. Par exemple, la nécessité de se laver avant d'entrer dans l'eau, comme le note Donald Richie : les japonais se font un plaisir d'apprendre aux étrangers les règles sacro-saintes du bain, sans doute pour bien se pénétrer de l'importance de la notion de purification, en amont de celle de la pure hygiène. Dans la pratique, cette règle reste souvent théorique, et n'est pas toujours respectée. Le point de vue adopté par Yoshiharu Tsuge, autre dessinateur de mangas, est assez différent, puisque l'un de ses personnages suspecte le bain d'avoir été souillé, précisément à cause d'un panneau d'interdiction. Sa perspective est réaliste, et il a trouvé son inspiration dans les auberges anciennes de villages reculés. Akutagawa Ryûnosuke, dans la nouvelle « L'Illumination créatrice » évoque un sentô de la fin de l'époque Edo il est vrai, mais fort peu hygiénique : «Les uns bavardaient, les autres chantonnaient, tous plongés dans l'eau chaude qui clapotait paresseusement et dont la surface

2. Le mot apparait en 1401 pour la première fois.

3. Critique, « Dans le bain japonais », $\mathrm{n}^{\circ} 428-429,1983$. 
était irisée par la graisse humaine qui y fondait sous les rayons troubles venus de l'entrée de la "grenade". Les forts effluves piquaient le nez ${ }^{4}$. » En fait, l'origine du bain traditionnel japonais remonte à l'époque Edo. Le système décrit ici, la grenade, ou zakuroguchi, consistait en une entrée qui capturait l'air et les vapeurs chaudes sans bouger les portes coulissantes. Il faut distinguer ici les bains de la ville d'Edo, organisés autour d'un grand bassin d'eau chaude, nommé yuya (湯屋, boutique d'eau chaude), alors qu'à Osaka, les bains publics étaient des bains de vapeur avec un bassin peu profond, nommés mushiburo (蒸し風呂, ou bain de vapeur). Le second système produisait une atmosphère vaporeuse et obscure. Il fut abandonné à l'ère Meijis .

\section{La dimension sacrée de la pratique des bains}

Le Japon ancien aurait été marqué par d'innombrables rites de purification pratiqués par l'aristocratie. Encore maintenant, on place un petit tas de sel sur le pas de la porte. Une explication est que, dans les zones montagneuses, l'aspersion d'eau de mer étant impossible, on la remplaçait par le sel. Mais l'on retrouve cette pratique partout, y compris sur le littoral. Un manquement aux tabous d'impureté serait la seule marque de péché au Japon. Les bains naissent dans l'enceinte du temple bouddhique. Chaque année depuis 753, le Bouddha du Todai-ji de Nara est purifié à l'emplacement des bains anciens. Seyoku est un bain de charité pour les fidèles dont on ne trouve pas l'équivalent dans les pays voisins, Chine ou Corée. Les bains curatifs deviennent des lieux de culte, et furent pratiqués ensuite par les gens ordinaires.

À l'origine de la pratique du bain japonais, le discours officiel contemporain avance un souci de purification, comme un exorcisme de l'impureté. On se débarrasse des souillures de la journée avant le sommeil. La purification rituelle par l'eau se nomme yuami, ou misogi (裙). Un bain rituel de purification était en principe considéré comme nécessaire après un enterrement. Le souci des ablutions et de la lustration est lié aux croyances locales, nommées shinto, avant l'introduction du bouddhisme, et serait peut-être à l'origine de la passion hydrothérapique des Japonais. Du moins, la dimension ésotérique et religieuse ancienne constitue un arrière-plan à la pratique inconsciente, ou insouciante des Japonais aujourd'hui. Mais un lien de cause à effet entre croyances religieuses et pratique profane est impossible à affirmer avec certitude. La thalassothérapie est joyeuse à l'origine, comme il l'est raconté dans un texte ancien au sujet de la rivière Tamatsukigawa. Mettre fin aux maladies, développer la beauté physique, tels sont les avantages que les anciens pensaient tirer du bain dans l'eau

4. Ryûnosuke Akutagawa, Rashômon et autres contes, Paris, Gallimard/Unesco, 1965, p. 169.

5. Scott Clark, Japan, a view from the bath, Hawaï, University of Hawaï press, 1994, p. 28 sq. 
divine. Le dignitaire d'Izumo s'y rend lui-même pour un banquet tapageur. L'eau sacrée enlève les souillures. De plus, elle régénère le corps. Il s'agit de rafraîchir le centre spirituel (kokoro $)^{6}$.

Une grande fête se nomme nemuri nagashi (« mise à l'eau de la somnolence »), précédée et suivie de rites de purification ${ }^{7}$. Les participants expulsent les souillures qui risqueraient d'entraver le déroulement des travaux agraires à la saison chaude, sous le triple visage de la somnolence, des insectes et de la maladie. Le thème de l'expulsion de la somnolence domine les préparatifs du bon (la fête des morts, qui se déroule en été). La baignade est alors une pratique de purification très répandue. Le but est de chasser les démons du sommeil en jetant dans l'eau l'état somnolent.

Le sept juillet, les enfants se jettent dès l'aube, ou le plus tôt possible, dans la rivière, pour ne pas ramasser la somnolence de ceux qui se seraient déjà baignés. L'enfant risquerait alors d'être un lève-tard pour l'année entière.

\section{Le bain domestique (o-furo) : les fonctions d'accueil et de relation}

Mizubayashi précise bien que l'o-furo (お風呂) fait partie du rite de l'accueil : la proposition de prendre un bain n'est pas à proprement parler un conseil d'hygiène, mais bien une invitation au bien-être qui naît au contact de l'eau chaude. Le fait de marcher sans chaussures et même sans mules (sur les tatamis) n'est pas étranger à une même progression dans l'intimité de l'espace domestique. Une fois évacuée la question fonctionnelle de l'hygiène, tremper dans l'eau devient une activité en soi. En effet, on ne se lave pas dans la baignoire, mais sur le sol carrelé, à l'aide d'une bassine, et grâce à une robinetterie étudiée. Une fois savonné et rincé, on entre dans la baignoire, déjà propre en somme. Le bain devient source de jouissance et délectation esthétique. Il s'agit moins de nettoyer que de donner une nouvelle fraîcheur au corps et à l'esprit, dans une recherche qualitative de sensations agréables. L'expression sappari shita (se sentir régénéré, ou rafraîchi) indique cette nuance mais plutôt lors d'une promenade dans la nature. Pour le bain, kimochi ga ii (éprouver un état de bien-être) conviendrait. Le fait de partager une même salle d'eau dans une auberge traditionnelle (旅館, ryôkan) participe du plaisir de voyager en retrouvant une ambiance « familiale ».

Une fonction encore plus importante du bain serait celle d'établir des rapports interindividuels : le bain serait pris en compagnie de personnes avec qui on entretient des liens d'affection, avec le privilège d'une « relation immédiate ${ }^{8}$ ». Mizubayashi cite une scène d'un film d'Ozu Yasujiro de 1942, Chichiariki (Il était un père) où le fils annonce à son père sa décision

6. Scott Clark, op. cit., p. 5.

7. Laurence Caillet, Fêtes et rites des quatre saisons au Japon, Paris, POF, 1981, p. 315.

8. Akira Mizubayashi, «Autour du bain », Critique, op. cit., p. 12. 
de se marier, et de quitter la maison, dans l'eau du bain. Le bain est une habitude ancrée dans le corps des Japonais, suivant un souvenir d'enfance sans doute heureux : l'heure du bain'. En effet, en dehors de la gymnastique du fœtus, si l'on traduit le terme taikyô, fœtus lui-même parfois désigné comme l'《enfant des eaux》 (水子, mizuko, même si ce terme est plutôt réservé à l'avorton, l'enfant mort-né ou avorté), la vie du nouveau-né commence près d'une petite baignoire en plastique, le corps lavé restant entouré d'un linge de gaze. Mais dès le deuxième mois, le bain du bébé a lieu dans la baignoire familiale, avec le père, comme le veut la tradition. Ce sont donc de fréquents contacts corporels, et de minutieux astiquages (même le nombril n'y échappe pas). Aucun tabou n'existe devant la nudité corporelle, et la fille pré-pubère prend le bain avec son père, ou les autres membres de la famille, dans un renforcement des liens familiaux (hadaka no tsukiau, "relation tactile», ou skinship). La communication passe dans l'immédiateté du corps et des affects positifs ou négatifs (kimochi ga ii (bien-être); kimochi ga warui (malaise)), dans un abandon à la perception, sans réflexion intérieure, dans une simple répercussion affective. Cette éducation à la sensation passe notamment par le bain familial, et la relation affective de la mère avec le nouveau-né expliquerait, selon certains anthropologues, le rapport « immédiat » des Japonais avec la nature.

L'heure du bain est très attendue. Le goût lustral des Japonais viendrait de la petite enfance, un bain par jour en hiver, le soir, pour conserver la chaleur du corps, et comme moyen de prévention contre la grippe. Le bruit de l'eau qui se répercute sur les parois des salles de bain japonaises serait le seul rappel sonore authentique du passé. C'est aussi un facteur de transparence de la vie domestique : chacun dans le voisinage sait que l'autre respecte les mêmes usages, presque au même moment.

\section{Les bains publics : un lieu de sociabilité spécifique}

Avec la rue marchande et le temple, les bains sont le cour de tout quartier. Il faut noter l'absence en général de « lieux publics » au Japon, tels que les places ou agoras. Les bains publics sont liés à un mode de vie, qui est celui des quartiers populaires de shitamachi (la ville basse). Le sens du mot sentô, littéralement « eau chaude payante », serait l'expression la plus accomplie du souci de la propreté uni au sens de la vie communautaire.

\section{Origines et transformation des sentô (銭湯)}

Si l'on résume brièvement l'histoire des bains publics, leur origine remonterait au $\mathrm{VI}^{\mathrm{e}}$, ou au $\mathrm{VII}^{\mathrm{e}}$ siècle, avec le bouddhisme. Cette tradition fut

9. Jean-Claude Jugon, Petite enfance et maternité au Japon, Paris, L'Harmattan, 2002. Les Japonais utilisent le terme anglais " skinship » pour désigner la relation parents-enfants, en particulier dans le bain. 
rejetée seulement avec la confrontation à l'Occident, à l'ère Meiji, mais les rapport des Japonais avec les bains publics ont évolué lentement au cours du temps, avant la réaction, parfois négative, des étrangers. Les premiers bains publics véritables naissent à l'ère Heian, avec les machiyu (町湯) - littéralement, " eau chaude de la ville ». Le furoya-san est le «marchand de bain de vapeur ${ }^{10} »$. Au machiyu, les baigneurs vont abandonner le port du yukata blanc, et le silence liturgique pour la nudité, le rire et les bavardages. La séparation des sexes à partir de l'âge de sept ans, recommandée par les prescriptions confucianistes, n'est jamais appliquée. Quand l'Occident médiéval se replie sur ses inhibitions face à la nudité, le Japon trouve au contraire plaisir au bain. En Europe, l'aversion quasi-religieuse pour le bain entraîna un lavage exclusif des parties visibles du corps, dans une peur de l'infiltration qui prit fin avec le triomphe de l'hygiénisme au XIX ${ }^{\mathrm{e}}$ siècle.

Malgré la fin de la mixité des sentô, en raison de l'intervention pudibonde notamment des Anglo-Saxons au Japon, et par souci de soigner une image de décence et de civilisation, la même relation émotive et sociale que dans le bain familial s'y maintint. L'écrivain Shikitei Samba (17251822) a fondé sa gloire littéraire sur la description des bains publics à la fin du XVIII ${ }^{\mathrm{e}}$ siècle. Il a publié notamment Ukiyo-buro (traduit par "Au bain public », mais dont le sens est plus proche de « bain du monde flottant»), et Ukiyo-doko (traduit par « Chez le barbier », et qui serait plus exactement, " Chez le barbier du monde flottant »). Ce sont des documents pris sur le vif de la vie du petit peuple d'Edo. Cet auteur est cité par Akutagawa. Un conteur (落語家, rakugo-ka), Kokontei Shinshô (1890-1983) continuera à fréquenter les bains, parce qu'il considère cet univers comme inséparable de son art, et bien des dialogues réalistes inclus dans les récits proviennent sans doute de ce lieu d'échange et de conversation. La fin de la mixité n'eut lieu que sous influence étrangère, dans le quartier de Tsukiji, puis dans le port de Yokohama, à partir de 1862. Les allées et venues de personnes dévêtues semblaient suspectes, alors que les tireurs de pousse, les portefaix, charpentiers, et autres artisans travaillaient le plus souvent presque nus, revêtus seulement d'un cache-sexe, fundoshi (褌, longue étoffe passée dans l'entrejambe). L'interdit catégorique viendra en 1871, sous peine de fermeture. Une certaine insouciance devant la mixité rendait la mesure presque incompréhensible, surtout que les bains étaient assez sombres, et dotés d'une perche en bambou qui séparait le bain en deux. De plus, les baigneurs utilisaient souvent leur petite serviette de bain pour cacher les parties sexuelles au sortir de l'eau ou en entrant dans le bain.

10. On parle encore de gosen sentô, « le bain qui coûte cinq sen ». Le bain est parfois uniquement un bain de vapeur, tandis que $y u$ désigne le bain d'eau chaude, pris dans le yuya, cuve en bois ou en pierre. Les baignoires peuvent être faites d'un bois tendre (hinoki, sugi), longues ou rondes, l'essentiel étant de pouvoir s'y mettre jusqu'au cou, le cas extrême étant le goemon-buro, où la partie inférieure est en fonte, et repose sur des pierres, avec le foyer en dessous. Une claie en bois est alors nécessaire. 


\section{Organisation du sentô}

Le prix était modique : quelques zeni. La mixité s'accompagnait de certaines précautions, comme une presque complète obscurité, le port du fundoshi (褌) pour les hommes, et de la robe du dessous pour les femmes. La seule paille de riz dans des sacs était utilisée et non le savon d'importation, trop coûteux. Du sel servait au lavage des dents. Le tenugui (手拭, petite serviette) servait à se frotter, humide, puis une fois essoré, à se sécher, sans souci des courants d'air.

Encore aujourd'hui, l'enfant est accompagné par sa mère, et a donc accès à la partie réservée aux femmes, tandis qu'à la maison, le père prend le bain avec les enfants. Les alentours du sentô sont frappants, à cause de la présence de clients vêtus de yukatas (浴衣, kimono de sortie de bain), de jinbé (bermuda), qui marchent avec le bruit des geta (下駄, socques en bois), la cuvette avec serviette et savon à la main. Le caissier (ou la caissière) du sentô se situe en retrait de l'entrée, sur une estrade (bandai, 番台), et regarde en général une petite télévision. Il surveille les allées et venues, côté homme et côté femme. Les baigneurs se trouvent côte à côte par la disposition des robinets, et non séparés par des cloisons. Dans les bassins, ils peuvent entrer en contact. Ce sont des bains collectifs et publics au sens de la relation publique qui s'y établit, de la sociabilité qui s'y manifeste. L'utilisation de l'espace est encore une fois bien différente au Japon, suivant une proxémique particulière. La description d'Akutagawa donne une idée de la diversité des conditions de ceux qui sont réunis. La clientèle apprécie ces moments où la frontière entre public et privé s'estompe. Ce n'est pas le cas de l'écrivain Bakin, qui est reconnu et tracassé dans le bain, tout comme l'est un personnage dans le roman Shiosai (Le Tumulte des flots) de Mishima. C'est donc un lieu hybride qui ne relève ni totalement du privé ni totalement du public, situé de manière indécise entre les deux.

\section{Les stations thermales (onsen, 温泉) ${ }^{11}$}

Les stations thermales coïncident parfois avec l'emplacement de sources sacrées à vertu curative dont l'emplacement était autrefois soigneusement conservé secret par certains guerriers, pour que leurs ennemis n'en aient pas connaissance. Les paysans aiment en particulier se baigner pour ces raisons curatives, et comme un rite séculaire pour rendre le corps plus résistant. Une nouvelle de Dazai Osamu, Belle enfant ${ }^{12}$ évoque le onsen de Yûmura, dans la province du Yamanishi, sous une chaleur étouffante.

11. Une autre dénomination est celle de Rotenburo, 露天風呂, bain à l'air libre, (ou Iwaburo, 岩風呂, bain dans les rochers), sources naturelles d'eau chaude qui jaillissent du rocher, et qui n'ont pas été captées. On peut ainsi se baigner au milieu du manteau neigeux. Philippe Pons cite un onsen encore mixte, dans le Tohoku resté plus simple et charmant, en insistant sur son caractère ni érotique ni grivois.

12. Dazai Osamu, Cent vues du Mont Fuji, Arles, Picquier, 1998. 
La relative déception du narrateur est compensée par la présence parmi les vieillards, comparés à des blaireaux apeurés, d'une jeune fille à la beauté semblable à une perle, ou à une pêche verte. Son extase esthétique devant ce corps qui brille par contraste avec les autres corps décrépits n'a rien de lubrique. Le narrateur admire en particulier ses seins, et insiste sur l'absence de honte de la jeune fille. Il la rencontre un autre jour chez le coiffeur du village, hésitant à lui confier : « Vos seins me sont plus familiers que votre visage. » Il la salue, et songe au lien quasi-familial qui les lie. L'activité du regard mérite un traitement particulier dans sa relation aux arts visuels et à la description littéraire.

\section{Le regard esthétique et curieux dans le bain public}

C'est un exemple d'un certain exercice du regard qui n'est pas voyeuriste au sens strict du terme, mais qui repère les différences, et goûte pleinement le privilège du mélange social, de la diversité des conditions sociales et humaines réunies pour l'occasion. C'est ce regard qui motive la description de Shikitei Samba, sur laquelle se greffe l'art d'Akutagawa Ryûnosuke.

Les récits dessinés par Yoshiharu Tsuge ne sont pas avares de ce type de notation, puisque l'on voit un samouraï qui révèle malgré lui une longue estafilade dans le dos, sans vouloir dire son nom. Celui qui est réuni par le hasard avec lui dans le bain soupçonnera qu'il est un grand samouraï, tandis que ce dernier veut cacher cette cicatrice qui est le vestige d'une erreur technique au combat, voire de sa lâcheté. Un autre récit du même dessinateur montre un dos tatoué d'étrange manière qui interpelle un compagnon de bain. Les corps tatoués qui émergent de l'eau des bains publics paraissent revêtus de costumes de bain 1900. Leur style est étroitement lié aux images du monde flottant (Ukiyo-e). On les appelle Nishiki hada (錦絵 肌) ou « peaux de brocart ${ }^{13} »$.

Il s'agit de lire le corps de l'autre, et de façon quasi-exclusive au bain, une identité parfois criminelle (tatouage en signe d'opprobre, et de châtiment, soit irezumi no kei). L'origine de la mode des tatouages est le monde de l'estampe qui se plaît à représenter des brigands tatoués. Mais les estampes des derniers grands maîtres décadents, comme Kunisada, Eisen, ou Kuniyoshi, sont autant le reflet que l'origine de la richesse des motifs et des couleurs des tatouages de l'époque. Le bain public devient un lieu d'exhibition. On organise même des concours de tatouages. L'autre véhicule de la présence des tatouages étant le théâtre Kabuki, avec le type du criminel de charme (Iro-aku). Les vedettes de Kabuki se faisaient d'ailleurs souvent tatouer. On appelle encore les tatouages les fleurs d'Edo (Edo hana) en raison de leur beauté. La naissance de la vocation d'un tatoueur peut être la vision de tatoués au bain, comme ce fut le cas pour Horichô, né 
en 1931 à Kumamoto. La fréquentation de prostituées tatouées peut être un autre déclencheur. On peut évoquer encore le cas de la vocation de Takagi Akimitsu (né en 1912), fasciné par le tatouage porté par une femme aperçue aux bains, alors qu'il y était allé à l'âge de cinq ou six ans, accompagné par sa mère.

Le lien avec la prostitution est assez évident, dans la proximité des ateliers de tatouage avec l'ancien quartier réservé de Yoshiwara, à Asakusa. Les pensionnaires étaient souvent tatouées. Pour autant, les tatoués ne sont pas des voyous en général, mais des gens ordinaires qui veulent acquérir des pouvoirs supplémentaires, de l'aveu même de certains tatoueurs, comme Horichô, encore en activité à Asakusa au début des années $1990^{14}$. À l'époque Edo, il s'agissait, en dehors des simples voyous ou malfrats, des portefaix, ou bien des sapeurs-pompiers (tobi). En prison, les tatoués ne pouvaient pas se baigner avec les autres prisonniers, de peur de l'ascendant qu'ils risquaient de prendre sur les autres, et cette interdiction existe dans certains sentô ou saunas, mais elle n'est pas toujours appliquée. Les forces de l'ordre s'intéressent toujours aux rassemblements de tatoués. En résumé, si le tatouage est lié à l'histoire des voyous et de la prostitution, comme ailleurs, la majorité des hommes tatoués sont plutôt des artisans, des forains, des petits commerçants, des camionneurs, des pêcheurs, ou jadis, des mineurs. En somme, ces tatoués visibles notamment dans les bains, étaient plutôt des gens ordinaires.

\section{Le bain, lieu d'une construction imaginaire}

Lieu de jouissance physique, le bain favorise aussi une extase poétique, qu'illustre parfaitement encore la nouvelle d'Akutagawa Ryûnosuke, "L'illumination créatrice ». En effet, Takigawa Bakin, l'écrivain en mal d'inspiration, et bourreau de travail, une fois dans le bain très chaud du Matsu no ŷu, est sujet d'une vision qualifiée de " romantique », et plus précisément une scène de son roman. Un vaisseau se déplace sur une mer couverte de brume. Sur quel support se lève cette rêverie? Non pas sur le décor du mur du fond, en général un paysage touristique en carreaux de faïence, mais sur un écran souple et propice à toutes les métamorphoses, la vapeur du bain.

C'est au pied de la lettre le fonctionnement de l'imagination qui est saisi, tel qu'il a pu être défini en Occident : " And as imagination bodies forth / The forms of things unknown, the poet's pen / Turns them to shapes, and gives to airy nothing /A local habitation and a name ${ }^{15}$. "

Ce qui est aussi indiqué dans une illustration de Suehiro Maruo à propos du bain, et encore dans une autre histoire du même dessinateur,

14. Philippe Pons, op. cit., p. 80.

15. William Shakespeare, A Midsummer Night's dream, acte V, scène I, 14-17. 
où le contexte franchement surnaturel et érotique, favorise l'émergence d'un visage monstrueux de spectre dans la vapeur du bain. Car le bain n'a pas la transparence des dessins de Suehiro Maruo. Le sentô en particulier est un lieu décrit comme obscur par Akutagawa Ryûnosuke, encombré de vapeurs : « une buée plus épaisse que brouillard alourdissait l'air ${ }^{16}$ ». Il faut noter que l'art japonais n'aurait pas atteint certains sommets dans la représentation du nu sans l'existence de bains mixtes, comme le suggère sur un mode cocasse le récit de Nosaka intitulé Le Moine-Cigale. Précisément, l'un des aspects importants de la construction imaginaire est l'élaboration de fantasmes sexuels, parfois sadiques, qui sont souvent déçus, et leur expression littéraire ou graphique. À cet égard, Mizubayashi note que, dans le cinéma européen, le thème de la salle de bain conduit immanquablement à l'érotisme, et au meurtre. Qui faut-il croire? D'un côté, nous avons une littérature de veine populaire et picaresque, allant de Jippensha Ikkû ( $A$ pied sur le Tokaido) à Nosaka Akiyuki (Les Pornographes, Le Moine-Cigale) qui prend le prétexte de la nudité et de la promiscuité des bains (ou des salles de massage) pour exprimer les réalités sexuelles dans l'innocence la plus entière; de l'autre, sans doute sous influence occidentale, un Suehiro Maruo développe la salle de bains. Une des dimensions de l'érotisme nippon, qui est le voyeurisme, satisfait dans les Nozokibeya ( cabines de peep show »), semble se donner peu de carrière à l'occasion du bain, sinon dans un film de Suzuki Seijun (Mémoires d'un tatoué), et encore de façon extrêmement chaste et esthétisée, en vue de sculpter une femme en figure salvatrice de Kannon. La disposition des bains profonds ne favorise guère le voyeurisme. Tanizaki fait d'une simple baignoire le lieu d'une expérience cardinale, et le prétexte au développement de divers fantasmes, dans le cadre de la maison conjugale décrite dans Kagi (Confession impudique).

Mais la réalité semble autre, tout au moins celle des bains publics, à l'exception d'établissements spécialisés. Donald Richie, qui a vécu un demi-siècle au Japon, affirme : «Dans ce pays, il y a un endroit et un moment pour chaque chose, et le sexe n'a pas sa place aux bains ${ }^{17}$. » Selon lui, on ne trouverait pas de voyeurs aux bains.

\section{Établissements de bain et quartiers de plaisir}

Il existe toutefois une proximité historique et réelle entre établissements de bains et chaya, ou maisons de rendez-vous. Les prostituées étaient des clientes assidues des établissements de bain. Une loi anti-prostitution fut finalement adoptée en 1958, et l'ancien Yoshiwara anticipa sa disparition en se convertissant en lieu d'accueil d'établissements de bains à partir de 1957. La police lutta contre les « bains turcs » sauvages, salons de massage hydrothérapiques qui servaient de couverture à la prostitution non officielle.

16. Akutagawa Ryûnosuke, Rashômon et autres contes, op. cit., p. 169.

17. Donald Richie, Tôkyô, Paris, Autrement, 2000, p. 69. 
Les bains turcs, bagno toruko, abrégé en toruko, ou en koruto, nommés aussi rabuŷu (de l'anglais love prononcé à la japonaise, avec le japonais $y \hat{u}$, eau chaude) ont été remplacés par des sôpurando (ソープランド, de l'anglais soap et land, " pays du savon ») sur plainte d'un diplomate turc en 1984, et après un sondage d'opinion, mais s'est-on vraiment interrogé sur l'acclimatation du premier terme, et sur l'imaginaire qui a présidé à cette dénomination? Quelle était la représentation exacte que pouvaient se faire les Japonais de la réalité des bains en Turquie? Le bain turc est aussi nommé bain de vapeur. Il est présenté comme thérapeutique et convivial ${ }^{18}$. En fait, l'hygiène est l'un des préceptes de la foi islamique, suivant le hadith : « La propreté fait partie de la foi ». C'est l'obligation coranique la plus respectée. Il entre en composition dans la foi des fidèles. Les bains publics sont des waqfs, des biens donnés à la collectivité, dont les revenus vont à des fonds religieux ou de bienfaisance. Construire un hammam est un geste pieux, et l'on dit qu'une ville ne peut être parfaite sans un hammam. L'expression bain turc est synonyme de beauté et de splendeur, tout à l'opposé de l'usage japonais, ignorant du suprême raffinement de la civilisation ottomane.

Les toruko renvoient lointainement aux furoya de l'époque Edo, où lavage et frictions basculaient facilement vers d'autres services. Un senryu, qui est un poème satirique dans le style du haïku, affirme : "Avant les jours de congé, quand on décide de sortir ensemble, ça commence souvent au bain public. » Les employés des maisons de commerce, la veille de ces rares jours de congé, prenaient au bain la décision d'aller le lendemain faire la fête à Yoshiwara. Les bains publics étaient un lieu de détente et de plaisir. Les laveurs étaient nommés sansuke (三助), et il y avait des frotteurs, hommes ou femmes. Ces dernières étaient nommées yuna (湯女), d'après la désignation de la servante de maison. Le lavage, nagashi, pouvait donner lieu à d'autres prestations ensuite. Le saké se prenait au premier étage, ou le sakurayu (infusion de fleurs de cerisier).

Le plaisir pouvait se poursuivre dans une chaya du voisinage. Des laveurs existaient encore dans les années 1960, mais les yuna furent interdites à la fin de l'époque Edo. Les yuna sont aussi appelées kami arai onna, les femmes qui lavent les cheveux. La dimension ambiguë du lavage a été récupérée dans les sôpurando.

Une autre composante de l'imaginaire du bain est celle de l'enfer (Jigoku, 地獄), ou du mauvais lieu (akusho, 悪所), dans une lecture de la chaleur comme éventuelle souffrance, comme sous l'effet d'une punition. D'ailleurs, le châtiment infligé au bandit Ishikawa Goemon (石川 五右衛門), dans une baignoire en hauteur avec un feu en dessous, était une torture des plus ordinaires sous les Tokugawa, sur le terrain d'exécution de Kazukahara, actuellement près du quartier de San-ya, à Tôkyô. Le condamné était torturé par immersion dans une cuve d'eau bouillante.

18. Pascal Meunier, Hammams : les bains magiciens, Paris, Dakota, 2000. 


\section{Sortie de bain}

Selon Philippe Pons, on retrouverait dans le jeune théâtre des années 1960, dans les pièces de Kara Jurô en particulier, le bain public comme métaphore de toute la culture japonaise. En somme, fréquenter le Japon, c'est fatalement s'exposer, un jour ou l'autre, à entrer dans le bain. On n'en sort pas. La visualisation du temps infini se ferait dans la culture japonaise suivant la ligne droite et le cercle. On peut rejoindre la Terre Pure en respectant les prescriptions de la Jôdo-shinsh $\hat{u}$ (浄土真宗), tandis que pour les sectes Zen (Rinzai-shû, 臨済宗, Sôtô-shu, 曹洞宗), le but ultime est de connaître le Satori (悟り, expérience mystique), dans la fin de la distinction entre soi et autrui, entre l'instant et l'éternité. Précisément, le bain public japonais (ou sa version domestique) permet une expérience voisine de ces états mystiques. L'âme se nettoie de la pollution du monde extérieur : furô wa tengok $u^{19}$. Plus simplement, et sans grande difficultés, l'expérience du bain japonais ne donne-t-elle pas l'occasion d'une expérience profane du même état?

Junko Komatsu

19. «Le bain, c'est le paradis.» 